\title{
ON DECOMPOSABILITY OF COMPACT PERTURBATIONS OF OPERATORS
}

\author{
M. RADJABALIPOUR
}

ABSTRACT. Let $A$ be a Hilbert-space operator satisfying the growth condition $\left\|(z-A)^{-1}\right\| \leq \exp \left\{K[\operatorname{dist}(z, J)]^{-s}\right\}, z \notin J$, where $J$ is a $C^{2}$ Jordan curve, and $K>0, s \in(0,1)$ are two constants. Let $T=A+B$

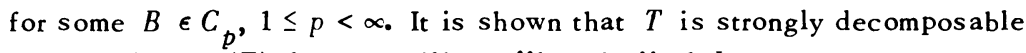
if and only if $\sigma(T)$ does not fill the "interior" of $J$.

1. H. Radjavi and the author [13] showed that if a Hilbert-space operator $T$ is the sum of a unitary operator $A$ and an operator $B$ of a Schatten class $C_{p}(1 \leq p<\infty)$ such that $\sigma(T)$ does not fill the unit disc, then $T$ is strongly decomposable. In the present paper we show that the above statement remains true if

(a) the unit disc is replaced by a domain whose boundary is a $C^{2}$ Jordan curve $J$,

(b) the unitary operator is replaced by an operator $A$ whose resolvent satisfies the growth condition

$$
\left\|(z-A)^{-1}\right\| \leq \exp \left\{K[\operatorname{dist}(z, J)]^{-s}\right\}, \quad z \notin J,
$$

where $s \in(0,1)$ and $K>0$ are two constants. (By $S^{-1}$ we mean it exists and is bounded.)

(Recall that (i) an invariant subspace $Y$ of $T$ is called a maximal spectral subspace of $T$ if $M \subseteq Y$ for all invariant subspaces $M$ of $T$ such that $\sigma(T \mid M) \subseteq \sigma(T \mid Y)$, (ii) $T$ is said to be decomposable if for every finite open covering $G_{1}, G_{2}, \ldots, G_{n}$ of $\sigma(T)$ there is a family $Y_{1}, Y_{2}, \ldots, Y_{n}$ of maximal spectral subspaces of $T$ such that $\sigma\left(T \mid Y_{i}\right) \subseteq \bar{G}_{i}, i=1,2, \ldots, n$, and $H=Y_{1}+Y_{2}+\cdots+Y_{n}$ where $H$ is the underlying Hilbert space, (iii) $T$ is said to be strongly decomposable if the restriction of $T$ to an arbitrary maximal spectral subspace of $T$ is again decomposable.)

Let us begin with a detailed introduction. Many authors have proved the existence of a nontrivial invariant subspace for the perturbation of a Hermitian operator $A$ by an operator $B$ of a class $C_{p}(1 \leq p<\infty)$; a complete list of these authors is given in $\left[6, p_{0}\right.$ 2122]. These results were further generalized by $\mathrm{K}$. Kitano [8] to the case where $A$ is a normal operator

Received by the editors September 9, 1974.

AMS (MOS) subject classifications (1970). Primary 47A 15, 47B05, 47B15.

Key words and phrases. Hilbert space, bounded operator, invariant subspace, growth condition, compact operator. 
with spectrum on a $C^{2}$ Jordan curve (see also [14, Chapter 6] in this direction) and by $C$. Apostol [1, Theorem 3.5 and its proof] to the case where the resolvent of $A$ satisfies the growth condition

$$
\left\|(z-A)^{-1}\right\| \leq K[\operatorname{dist}(z, J)]^{-n}, \quad z \notin J,
$$

where $J$ is a $C^{2}$ Jordan curve and $K, n$ are positive constants. I. Colojoară and C. Foiaș $\left[3\right.$, Theorem 5.5.2, p. 166] showed that if $T^{*} T-I \in C_{p}$ $(1 \leq p<\propto)$ and $\sigma(T)$ lies on the unit circle, then $T$ is strongly decomposable (actually they showed that $T$ is an $N$-unitary operator which is much stronger); the author [12, Corollary 3] proved that if $T$ is the sum of an operator $A$ satisfying (2) and an operator $B \in C_{p}(1 \leq p<\infty)$, and if $\sigma(T) \subseteq J$, then $T$ is strongly decomposable. A. Jafarian [7] showed that if $0 \leq I-T^{*} T \epsilon$ $C_{1}$ (the trace class) and $\sigma(T)$ does not fill the unit disc, then $T$ is still strongly decomposable (see also B. Sz.-Nagy and C. Foias, [16, Theorem VIII. 3.1, p. 337]). More generally, H. Radjavi and the author [13] proved that if $\sigma(T)$ does not fill the interior of the unit disc then the condition $T^{*} T-$ $I \in C_{p}(1 \leq p<\propto)$ is sufficient for strong decomposability of $T$. Actually we proved more; if $\sigma(T)$ does not fill the "interior" of a $C^{2}$ Jordan curve $J$ and if, for any hyperinvariant subspace $M$ of $T, T \mid M$ (respectively $\left.\left(T^{*} \mid M^{\perp}\right)^{*}\right)$ is the sum of a normal operator having spectrum on $J$ and an operator of class $C_{p}(1 \leq p<\infty)$, then $T$ is strongly decomposable. As a generalization of these results we prove

Theorem 1. Let $A$ satisfy the growth condition (1). Let $B \in C_{p}$ for some $p \in[1, \infty)$ and let $T=A+B$. Then $T$ is strongly decomposable if and only if $\sigma(T)$ does not fill the interior of $J$.

The proof is given in the next section. As immediate corollaries we have:

Corollary l. If $T$ is the sum of a normal operator with spectrum on a $C^{2}$ Jordan curve $J$ and an operator of class $C_{p}(1 \leq p<x)$, then $T$ is strongly decomposable if and only if $\sigma(T)$ does not fill the interior of $J$.

Corollary 2. Let $T$ be as in Theorem 1. Assume $\sigma(T)$ has more than one point. Then $T$ has a nontrivial hyperinvariant subspace.

2. We prove Theorem 1 in a series of propositions and lemmas. All operators are bounded and defined on a Hilbert space.

Notations and definitions. Let $B(H)$ be the space of all bounded linear operators defined on a Hilbert space $H$.

(a) An operator $T \in B(H)$ is said to have the single valued extension property if there exists no nonzero $H$-valued analytic function $f$ such that $(z-T) f(z) \equiv 0$. 
(b) $\bar{G}=$ the closure of a set $G \subseteq \mathbf{C}$.

(c) $C_{p}$ is the ideal of compact operators $T$ such that $\Sigma\left(\mu_{n}\right)^{p}<\infty$, where $\mu_{1}, \mu_{2}, \ldots$ are the eigenvalues of $\left(T^{*} T\right)^{1 / 2}$ arranged in decreasing order and repeated according to multiplicity for $0<p<\infty ; C_{\infty}$ is the ideal of all compact operators.

The following proposition is known.

Proposition 1. Let $T \in B(H)$ be the sum of an invertible operator $A$ and a compact operator $B$. Assume $\sigma(T)$ fills a neighbourbood of the origin. Then $T$ is not decomposable.

Lemma 1. Let $\phi$ be a conformal mapping from the unit disc onto the "interior" of a $C^{2}$ Jordan curve $J$. Then there exist two positive constants $K_{1}$ and $K_{2}$ such that

$$
K_{1}(1-|z|) \leq \operatorname{dist}(\phi(z), J) \leq K_{2}(1-|z|) \quad \text { for }|z|<1 .
$$

Proof. The first inequality follows from the Koebe-Bieberbach one quaro ter theorem [18, Theorem III. 40, p. 85] (see also [15, proof of Theorem 8, p. 143] for more details); the second inequality follows from [18, Chapter IX, $\$ 3$, Lemma 1, p. 359] together with the fact that $\phi^{\prime}(z)$ exists and is uniformly bounded for $|z| \leq 1$ [18, proof of Theorem IX.7, p. 361].

The following proposition is central to our investigations. First we need some definitions.

Let $B \in C_{p}$ for some positive integer $p$ and let $x, y$ be two arbitrary unit vectors in the underlying Hilbert space $H$. Let

$$
D_{0}(B)=\prod_{i}\left(1-\lambda_{i}\right) \exp \left(\sum_{j=1}^{p-1} j^{-1}\left(\lambda_{i}\right)^{j}\right), \quad D_{1}(B)=D_{0}(B)\left((I-B)^{-1} x \mid y\right),
$$

where $\lambda_{1}, \lambda_{2}, \cdots$ are the eigenvalues of $B$, numbered according to decreasing values of their moduli and repeated according to their multiplicities. Define $\|B\|_{p}=\left[\Sigma\left(\lambda_{i}\right)^{p}\right]^{1 / p}$ if $B$ is positive, and $\|B\|_{p}=\left\|\left(B^{*} B\right)^{1 / 2}\right\|_{p}$ in general. It is shown in [5, Lemma XI. 9.22 and Corollary XI. 9.25, p. 1106 and 1112] that if $1 \notin \sigma(B)$, then $\ln \left|D_{i}(B)\right| \leq \Gamma\left(\|B\|_{p}\right)^{p}, i=0,1$, where $\Gamma$ is a positive number depending only on $p$. (See also [10, Lemma 1].) Moreover, if $S$ is an arbitrary operator on $H$, then $\|S B\|_{p} \leq\|S\|\|B\|_{p}$ and $\|B S\|_{p} \leq\|S\|\|B\|_{p}$.

Proposition 2. Let $T$ be as in Theorem 1 and let $\sigma(T)$ not fill the "interior" of J. Let (the open set) G be either the "interior" or the "exterior" of $J$. Let $J_{1}$ be a nontrivial closed subarc of $J$. Let $M$ be an invariant subspace of $T$ such that $[G \cap \sigma(T \mid M)]^{-} \cap J_{1}=\varnothing$. Then there exist a constant $C>0$ and an open neighbourbood $\Delta$ of $J_{1}$ such that 


$$
\left\|(z-T \mid M)^{-1}\right\| \leq \exp \left\{\exp \left[C \operatorname{dist}(z, J)^{-s}\right]\right\}
$$

for $z \in \Delta \cap G$.

Proof. Assume without loss of generality that $p$ is a positive integer. Let $\Delta$ be an open neighbourhood of $J_{1}$ such that $\bar{\Delta} \cap G$ contains no points of $\sigma(T \mid M)$ and the boundary of $\Delta \cap G$ is a $C^{2}$ Jordan curve (containing $J_{1}$ ). Let $\phi$ be a conformal mapping from the unit disc onto $\Delta \cap G$. Let $x, y$ be two unit vectors in $M, f(z)=\left((z-T \mid M)^{-1} x \mid y\right)$ for $z \in \Delta \cap G$, and $g(t)=$ $f(\phi(t))$ for $t \in \mathbf{C}$ and $|t|<1$. Let

$$
T(r)=\frac{1}{2 \pi} \int_{0}^{2 \pi} \ln \left|g\left(r e^{i \theta}\right)\right| d \theta, \quad 0 \leq r<1
$$

be the Nevanlinna's characteristic function, of $g[11, \mathrm{p} .12]$, where $\ln ^{+} \alpha=$ $\max \{0, \ln a\}$ for $\alpha>0$, For $z \notin \sigma(T)$ and $z \in G \cap \Delta$ we have

$$
f(z)=\left((z-T)^{-1} x \mid y\right)=\left(\left[I-B(z-A)^{-1}\right]^{-1} x \mid\left(\bar{z}-A^{*}\right)^{-1} y\right)=\delta_{1}(z) / \delta_{0}(z),
$$

where

$$
\delta_{0}(z)=D_{0}\left(B(z-A)^{-1}\right)\left[\phi^{-1}(z)\right]^{-m}, \quad \delta_{1}(z)=\delta_{0}(z) f(z),
$$

and $m$ is the order of the zero of $D_{0}\left(B(z-A)^{-1}\right)$ at $z=\phi(0)$. (In view of [14, Lemma 6.7] $\delta_{0}$, and consequently $\delta_{1}$, are analytic on $\left.\mathrm{C} \backslash J.\right)$ It follows from the paragraph preceding the proposition that for $z \notin \sigma(T)$,

$$
\ln ^{+}\left|\delta_{i}(z)\right| \leq \exp \left\{\Gamma_{1}[\operatorname{dist}(z, J)]^{-s}\right\} \leq \exp \left\{\Gamma_{2}\left|1-\phi^{-1}(z)\right|^{-s}\right\}, \quad i=0,1,
$$

where $\Gamma_{1}, \Gamma_{2}$ are positive numbers independent of $z$ (see also Lemma 1). Choose $r \in(0,1)$ such that $\phi\left(r e^{i \theta}\right) \notin \sigma(T)$ for all $\theta \in[0,2 \pi]$. This is possible because $\sigma(T) \backslash J$ is countable (see [13, Lemma 1]). Applying Jensen's formula $[22, \mathrm{p} .3]$ to $\delta_{0}(\phi(t))$ we obtain

$$
\begin{aligned}
\ln \left|\delta_{0}(\phi(0))\right|= & \frac{1}{2 \pi} \int_{0}^{2 \pi} \ln ^{+}\left|\delta_{0}\left(\phi\left(r e^{i \theta}\right)\right)\right| d \theta \\
& -\frac{1}{2 \pi} \int_{0}^{2 \pi} \ln +\left|\frac{1}{\delta_{0}\left(\phi\left(r e^{i \theta}\right)\right)}\right| d \theta-\sum_{k} \ln \frac{r}{\left|a_{k}\right|},
\end{aligned}
$$

where $a_{1}, a_{2}, \cdots$ are the roots of $\delta_{0}(\phi(t))$ in the disc $|t|<r$. Therefore

$$
\begin{aligned}
T(r) \leq & \frac{1}{2 \pi} \int_{0}^{2 \pi} \ln ^{+}\left|\delta_{1}\left(\phi\left(r e^{i \theta}\right)\right)\right| d \theta \\
& +\frac{1}{2 \pi} \int_{0}^{2 \pi} \ln ^{+}\left|\delta_{0}\left(\phi\left(r e^{i \theta}\right)\right)\right| d \theta-\ln \left|\delta_{0}(\phi(0))\right|
\end{aligned}
$$

or $T(r) \leq \exp \left\{\Gamma_{3}(1-r)^{-s}\right\}$ where $\Gamma_{3}>0$ is independent of $r$. Since the set $\left\{r \in[0, \overrightarrow{1}]: \phi\left(r e^{i \theta}\right) \notin \sigma(T)\right.$ for all $\left.\theta \in[0,2 \pi]\right\}$ is dense in $[0,1]$, we have 
$T(r) \leq \exp \left\{\Gamma_{3}(1-r)^{-s}\right\}$ for all $r \in[0,1]$. Thus by $[11, \mathrm{p} .140]$,

$$
\ln \left|g\left(r e^{i \theta}\right)\right| \leq \exp \left\{\Gamma_{4}(1-r)^{-s}\right\} \text { for } 0 \leq r<1 \text {, }
$$

where $\Gamma_{4}$ is independent of $r$ and $\theta$. Another application of Lemma 1 yields

$$
\ln ^{+}|f(z)| \leq \exp \left\{C[\operatorname{dist}(z, J)]^{-s}\right\} \text { for } z \in \Delta \cap G \text {, }
$$

where $C$ is a constant independent of $z$. (Note that $f(z)$ remains bounded off $J$.) Since $x, y$ were arbitrary, the proof of the proposition is complete.

Some of the techniques of the proof of Proposition 2 are inspired from the proof of Theorem 1 of [10].

Proof of Theorem 1. The necessity of the condition follows from Proposition 1. The proof of the sufficiency is the same as the proof of [13, Theorem 1] with a slight difference. This difference is in the proof of [13, Lemma 5] which is a step toward the proof of [13, Theorem 1]. The growth condition (3) of Proposition 2 is not exactly the same as the one needed in the proof of [13, Lemma 5]. Fortunately, a similar proof can be constructed by using some results of J. I. Ljubic and V. I. Macaev (see [9, Lemma 2.2.1, p. 119]).

Although we have solved Problem 1 of [13], the following conjecture of that paper remains unsettled.

Conjecture. If $T$ is the sum of a normal operator having spectrum on a $C^{2}$ Jordan curve and an operator of some class $C_{p}(1 \leq p<\alpha)$, then the same is true for the restriction of $T$ to any hyperinvariant subspace. (The conjecture is true for $p=\infty[13$, Theorem 2].)

General remark. In view of [6, Theorem XVI.5.18, p. 2162], a spectral operator of finite type having spectrum on a $C^{2}$ Jordan curve $J$ satisfies the growth condition (1).

\section{REFERENCES}

1. C. Apostol, On the growth of resolvent, perturbation and invariant subspaces, Rev. Roumaine Math. Pures Appl. 16 (1971), 161-172. MR 45 \#933.

2. J. W. Calkin, Two-sided ideals and congruences in the ring of bounded operators in Hilbert space, Ann. of Math. (2) 42 (1941), 839-873. MR 3, 208.

3. I. Colojoară and C. Foias, The theory of generalized spectral operators, Gordon and Breach, New York, 1968.

4. R. G. Douglas, Banach algebra techniques in operator theory, Academic Press, New York, 1972.

5. N. Dunford and J. T. Schwartz, Linear operators. Part II: Spectral theory. Selfadjoint operators in Hilbert space, Interscience, New York, 1963. MR 32 \#6181.

6. - Linear operators. III, Interscience, Ncw York, 1971.

7. A. A. Jafarian, Weak contractions of Sz.-Nagy and Foias are decomposable, Rev. Roumaine Math. Pures Appl. (to appear).

8. K. Kitano, Invariant subspaces of some non-selfadjoint operators, Tônoku Math. J. (2) 20 (1968), 313-322. MR 39 \#2001.

9. Ju. I. Ljubič and V. I. Macaev, Operators with separable spectrum, Mat. Sb. 56 (98) (1962), 433-468; English transl., Amer. Math. Soc. Transl. (2) 47 (1965), 89-129. MR $25 \# 2450$. 
10. V. I. Macaev, A method for the estimation of the resolvents of non-selfadjoint operators, Dokl. Akad. Nauk SSSR 154 (1964), 1034-1037 = Soviet Math. Dokl. 5 (1964), 236-240. MR 28 \#1495.

11. R. Nevanlinna, Le théorème de Picard-Borel et la théorie des fonctions méromorphes, Gauthier-Villars, Paris, 1929.

12. M. Radjabalipour, Growth conditions and decomposable operators, Canad. J. Math. 26 (1974), 1372-1379.

13. M. Radjabalipour and H. Radjavi, On decomposability of compact perturbations of normal operators, Canad. J. Math. (to appear).

14. H. Radjavi and P. Rosenthal, Invariant subspaces, Springer-Verlag, Berlin, 1973.

15. J.G. Stampfli, A local spectral theory for operators. III: Resolvents, spectral sets and similarity, Trans. Ame r. Math. Soc. 168 (1972), 133-151. MR 45 \#4182.

16. B. Sz.-Nagy and C. Foias,, Analyse harmonique des opérateurs de l'espace de Hilbert, Masson, Paris; Akad. Kiadó, Budapest, 1967; English rev. transl., NorthHolland, Amsterdam, American Elsevier, New York; Akad. Kiadó, Budapest, 1970. MR $37 \# 778 ; 43 \# 947$.

17. A. E. Taylor, Introduction to functional analysis, Wiley, New York; Chapman \& Hall, London, 1958. MR 20 \#5411.

18. M. Tsuji, Potential theory in modern function theory, Maruzen, Tokyo, 1959. MR $22 \# 5712$.

DEPARTMENT OF MATHEMATICS, DALHOUSIE UNIVERSITY, HALIFAX, NOVA SCOTIA, CANADA 